\title{
Kantorovich's Inequality
}

\section{Morris Newman}

(August 18, 1959)

An elementary proof with a generalization of an inequality of Kantorovich is given.

Let $A$ be a hermitian positive definite matrix with smallest eigenvalue $\alpha$ and largest eigenvalue $\beta$. Then Kantorovich's inequality ${ }^{2}$ states that for all vectors $x$ of unit norm,

$$
(A x, x)\left(A^{-1} x, x\right) \leq \frac{1}{4}\left\{\left(\frac{\alpha}{\beta}\right)^{\frac{1}{2}}+\left(\frac{\beta}{\alpha}\right)^{\frac{1}{2}}\right\}^{2} .
$$

In this note we give an elementary proof of (1) which allows an easy generalization. The results obtained do not depend on the order of $A$ and this is accordingly left unspecified.

We assume only that $A$ is hermitian, and that the eigenvalues of $A$ are contained in the closed interval $m \leq t \leq M$.

Let $f(t), g(t)$ be real functions such that

$$
\begin{gathered}
0<f(t), g(t)<\infty \quad \text { for } \quad m \leq t \leq M, \\
f(t), g(t) \text { are convex } \quad \text { for } \quad m \leq t \leq M .
\end{gathered}
$$

Then (2) implies that the matrices $F=f(A)$, $G=g(A)$ are well-defined and are hermitian positive definite. We shall prove:

Theorem. Let $x$ be any vector of unit norm. Put

$$
K=(F x, x)(G x, x) .
$$

Then for every positive $c$,

$$
2 K^{\frac{1}{2}} \leq \max \left(c f(m)+\frac{1}{c} g(m), c f(M)+\frac{1}{c} g(M)\right) .
$$

If in addition $f(M)-f(m)$ and $g(M)-g(m)$ are of opposite sign, then

$$
2 K^{\frac{1}{2}} \leq r f(m)+\frac{1}{r} g(m)
$$

where

$$
r=\left(-\frac{g(M)-g(m)}{f(M)-f(m)}\right)^{\frac{1}{2}}
$$

1 The preparation of this paper was supported (in part) by the Office of Naval Research.

${ }_{2}^{2}$ L. V. Kantorovich, Functional analysis and applied mathematics, Uspekhi Math. Nauk 3, 89 (1948).
Proof. Since $A$ is hermitian, $A$ is unitarily equivalent to a diagonal matrix. We may therefore assume without loss of generality that $A=\left(a_{i} \delta_{i j}\right)$, where $m \leq a_{i} \leq M$. Then also $F=\left(f\left(a_{i}\right) \delta_{i j}\right)$ and $G=\left(g\left(a_{i}\right) \delta_{i j}\right)$. Suppose that the $i^{\text {th }}$ coordinate of $x$ is $x_{i}$ and set $t_{i}=\left|x_{i}\right|^{2}$, so that

$$
t_{i} \geq 0, \quad \sum t_{i}=1,
$$

$x$ being of unit norm. Then $K$ becomes

$$
K=\sum f\left(a_{i}\right) t_{i} \sum g\left(a_{i}\right) t_{i} .
$$

Let $c>0$ be arbitrary and rewrite $K$ as

$$
K=\sum c f\left(a_{i}\right) t_{i} \sum \frac{1}{c} g\left(a_{i}\right) t_{i} .
$$

Then

$$
\begin{aligned}
2 K^{\frac{1}{2}} & =2\left(\sum c f\left(a_{i}\right) t_{i}\right)^{\frac{1}{2}}\left(\sum \frac{1}{c} g\left(a_{i}\right) t_{i}\right)^{\frac{1}{2}} \\
& \leq \sum c f\left(a_{i}\right) t_{i}+\sum \frac{1}{c} g\left(a_{i}\right) t_{i} \\
& \leq \max \left(c f\left(a_{i}\right)+\frac{1}{c} g\left(a_{i}\right)\right),
\end{aligned}
$$

by (6).

We now consider the function

$$
u=c f(t)+\frac{1}{c} g(t), \quad m \leq t \leq M .
$$

Because of assumptions (2) and (3) and the fact that $c$ is positive, we have that $u$ is convex and positive for $m \leq t \leq M$. This implies that

$$
\max _{m \leq t \leq M} u=\max (u(m), u(M))
$$

which proves (4).

If in addition $f(M)-f(m)$ and $g(M)-g(m)$ are of opposite sign, the choice $c=r$ is permissible and makes

$$
r f(m)+\frac{1}{r} g(m)=r f(M) \frac{1}{r} g(M),
$$


which gives (5). This completes the proof of the theorem.

Kantorovich's inequality (1) is the case $f(t)=t$, $g(t)=\frac{1}{t}$ for which $r=(m M)^{-1 / 2}$ (which is permissible when $A$ is positive definite).

Ky Fan in a written communication to the author points out that the theorem can be generalized as follows:

Let $A$ be a hermitian matrix of order $n$ with all its eigenvalues contained in the closed interval $\alpha \leq t \leq \beta$. Let $x_{1}, x_{2}, \ldots, x_{k}$ be vectors in unitary $n$-space such that

$$
\sum_{i=1}^{k}\left\|x_{i}\right\|^{2}=1
$$

Let $f_{1}, f_{2}, \ldots, f_{m}$ be positive convex functions for $\alpha \leq t \leq \beta$.
If $c_{1}, c_{2}, \ldots, c_{m}$ are positive numbers satisfying $c_{1} c_{2} \ldots c_{m}=1$, then

$$
\begin{aligned}
\prod_{i=1}^{m}\left\{\sum_{j=1}^{k}\left(f_{i}(A) x_{j}, x_{j}\right)\right\}^{1 / m} & \\
& \leq \frac{1}{m} \max \left\{\sum_{i=1}^{m} c_{i} f_{i}(\alpha), \sum_{i=1}^{m} c_{i} f_{i}(\beta)\right\}
\end{aligned}
$$

Furthermore if $A$ is positive definite and $\alpha>0$, Kantorovich's inequality can be replaced by

$$
\left\{\sum_{i=1}^{k}\left(A x_{i}, x_{i}\right)\right\}\left\{\sum_{i=1}^{k}\left(A^{-1} x_{i}, x_{i}\right)\right\} \leq \frac{1}{4}\left\{\left(\frac{\alpha}{\beta}\right)^{\frac{1}{2}}+\left(\frac{\beta}{\alpha}\right)^{\frac{1}{2}}\right\}^{2} .
$$

Washington, D.C.

(Paper 64B1-17) 